\title{
Un moment spencérien aux origines de la psychologie « scientifique » française?
}

L'Hérédité psychologique de Théodule Ribot

\section{Régine Plas}

\section{OpenEdition}

Journals

Édition électronique

URL : http://journals.openedition.org/aes/280

DOI : $10.4000 /$ aes. 280

ISSN : 2258-093X

Éditeur

Laboratoire LISAA

Référence électronique

Régine Plas, "Un moment spencérien aux origines de la psychologie " scientifique » française ? ", Arts et Savoirs [En ligne], 4 | 2014, mis en ligne le 15 mai 2014, consulté le 19 avril 2019. URL : http:// journals.openedition.org/aes/280 ; DOI : 10.4000/aes.280

Ce document a été généré automatiquement le 19 avril 2019.

Centre de recherche LISAA (Littératures SAvoirs et Arts) 


\title{
Un moment spencérien aux origines de la psychologie " scientifique » française?
}

\author{
L'Hérédité psychologique de Théodule Ribot
}

\section{Régine Plas}

1 Cet article est paru en mars 2013, sous le titre " ¿Un momento spenceriano en los orígenes de la psicología "científica" francesa? La herencia psicológica de Théodule Ribot ", dans la Revista de Historia de la Psicología, n 34, 2013, p.9-24, et en ligne à l'adresse: http://www.revistahistoriapsicologia.es/revista/. Je remercie Juan Antonio Vera, Editor Ejecutivo de cette revue, ainsi que Noemí Pizarroso, coordinatrice de ce numéro spécial sur l'histoire de la psychologie française, pour m'avoir aimablement autorisée à publier la version française de cet article, avec quelques modifications mineures.

\section{Introduction}

2 On a pu parler d'un «moment anglais de la pensée française », qui débuta à la fin du Second Empire, moment durant lequel l'œuvre d'Herbert Spencer fut mise au tout premier plan ${ }^{1}$. La doctrine spencérienne eut, chez les philosophes français, d'ardents défenseurs, non moins que d'ardents détracteurs, et elle suscita de nombreuses controverses. Au tournant des $\mathrm{XIX}^{\mathrm{e}}$ et $\mathrm{XX}^{\mathrm{e}}$ siècles, elle tomba dans le discrédit, puis dans l'oubli. Spencer fut également, comme l'écrivit un contributeur de L'Année psychologique au moment de sa mort, "très à la mode en France comme psychologue ${ }^{2}$ ». Il y eut, en effet, un moment spencérien de la psychologie française mais, à y regarder de plus près, ce moment fut de courte durée et représenté surtout par un philosophe de formation, Théodule Ribot (1839-1916), régulièrement présenté par une certaine historiographie, comme le «fondateur » de la psychologie « scientifique » française. En effet, c'est d'abord en tant que disciple de Spencer que Ribot devint un acteur du débat philosophique de son temps. Toutefois, au début du $\mathrm{xx}^{\mathrm{e}}$ siècle, il semble, lui aussi, avoir pris ses distances vis-à- 
vis du philosophe anglais. Il y a donc lieu de questionner la fascination que l'œuvre de Spencer suscita chez le jeune Ribot et de nous demander pourquoi, et dans quel contexte, le fait de se revendiquer de Spencer a pu contribuer à lui permettre de promouvoir la psychologie qu'il défendait.

\section{Situation de la philosophie française dans les années 1860}

3 De nombreux auteurs, historiens ou philosophes, ont souligné que le $\mathrm{xIX}^{\mathrm{e}}$ siècle français est parcouru par l'opposition à la fois politique, religieuse et intellectuelle entre spiritualistes et positivistes. En y regardant de plus près, cet antagonisme est souvent décrit de façon quelque peu schématique mais il est présenté, et probablement perçu, comme irréductible par certains des contemporains de Ribot, tel Hippolyte Taine, dans son ouvrage de 1857 sur Les Philosophes français du XIXe siècle ${ }^{3}$. Il faut donc évoquer, ne serait-ce que brièvement, le contexte national dans lequel Ribot fut amené à publier ses premiers travaux, en nous attachant plus précisément à la seconde moitié du XIX ${ }^{\mathrm{e}}$ siècle.

4 En 1850, Louis-Napoléon Bonaparte, qui n'était encore que le premier président élu de la République française, ne s'opposa pas au vote d'une loi sur l'instruction publique, passée à la postérité sous le nom de Loi Falloux, du nom de son initiateur, Alfred de Falloux, ministre de l'Instruction publique et député du très conservateur parti de l'ordre, alors majoritaire au sein de l'Assemblée nationale. Cette loi instaurait, entre autres, un partage entre l'enseignement public et l'enseignement privé et, elle consacrait globalement la mainmise de l'Église catholique sur l'ensemble du système scolaire. Elle plaçait les instituteurs sous le contrôle des curés, qui pouvaient obtenir leur déplacement grâce à un simple rapport envoyé aux évêchés. L'agrégation de philosophie, jugée subversive, fut supprimée en 1852, en même temps que l'agrégation d'histoire. Notons que l'année précédente, le jury de ce concours, principalement composé de philosophes spiritualistes avait refusé de recevoir Hippolyte Taine, en raison, semble-t-il, de son argumentation d'allure trop spinoziste ${ }^{4}$. Ce fut le triomphe du « parti clérical », comme le dénonça Victor Hugo dans son célèbre discours de janvier 1850 contre la loi Falloux ${ }^{5}$. À la fin des années 1850, et au cours des années 1960, grâce en particulier à Victor Duruy ${ }^{6}$, on assista à une libéralisation de l'université et à une réaction anti-spiritualiste de la part de jeunes intellectuels qui se réclamaient du positivisme dont la diffusion avait été assurée par Émile Littré et ses partisans. La riposte des spiritualistes ne se fit pas attendre et aboutit, en 1863, à l'échec de la candidature de Littré à l'Académie française, ainsi qu'à celle de Taine l'année suivante et à la révocation d'Ernest Renan, titulaire de la chaire d'hébreu au Collège de France, qui venait de publier sa Vie de Jésus.

5 Cependant, si les spiritualistes représentaient toujours la philosophie officielle, occupaient les chaires à l'université et au Collège de France, siégeaient au jury d'agrégation, certains d'entre eux, conscients de la diffusion de l'esprit positif, et de la menace qu'il représentait pour leur doctrine, en appelaient, avec le philosophe Émile Beaussire, à un "spiritualisme libéral » ouvert à la science. C'est le cas, notamment, d'Elme-Marie Caro, élu contre Taine à l'Académie française en 1874, ou de l'oncle du futur psychologue Pierre Janet, Paul Janet, qui réforma les programmes de l'enseignement de philosophie au début de la Troisème République. 


\section{Quand Ribot rencontre Spencer}

6 Théodule Ribot, né en 1839, entre en 1862 à l'École normale supérieure. En 1865, après avoir échoué à l'agrégation de philosophie, il est nommé chargé de cours au lycée de Vesoul, une petite ville de l'Est de la France, «11 rues et 6000 habitants », ainsi qu'il l'écrit à son ancien condisciple Alfred Espinas ${ }^{7}$. En 1866, ayant obtenu l'agrégation, il est nommé professeur et reste à Vesoul, qu'il a préférée à la ville normande de Coutances qu'on lui avait, semble-t-il, également proposée, car écrit-il à Alfred Espinas en 1966, « ici du moins, pas d'Évêque, pas d'esprit clérical » ${ }^{8}$. Il y « lit beaucoup : des livres de science, du Taine, des positivistes. Je déguste Stuart Mill $»^{9}$. Il reçoit de Londres les Principles of Psychology de Spencer, dont il écrit : «J'ai quelque envie de le traduire. Ce volume coûte 20 fr. (1 volume) ; mais ce n'est pas trop cher puisqu'il s'agit d'un positiviste. $»^{10}$

7 La suite de sa correspondance témoigne de l'admiration qu'il porte aussitôt à Spencer. En 1867, il écrit : « [...] c'est un des ouvrages les plus originaux et les plus intéressants que je connaisse. C'est la psychologie étudiée à la manière positive ${ }^{11} \mathrm{cad}$. [sic] abstraction faite de la question de substance et en s'appuyant sur la physiologie $»^{12}$ Il traduit les Principes de psychologie de Spencer, d'abord pour son usage personnel puis avec l'intention de les publier, en même temps qu'il se "plonge jusqu'au cou dans le positivisme anglais »" Toutefois, alors que sa traduction est achevée et sur le point d'être publiée, Spencer lui annonce qu'il travaille à une seconde édition refondue et doublée et qu'il l'agrée comme traducteur de cette seconde édition. Par ailleurs, en 1868, Ribot se dit " persécuté par le clergé : accusé de scepticisme, panthéisme etc. l'aumônier a fait un prône contre moi $»^{14}$. Il se rend à Londres la même année pour rencontrer Spencer, mais ce dernier est en vacances et Ribot annonce à Espinas qu'il va peut-être publier un article anonyme sur Spencer dans la Revue de philosophie positive de Littré. On ne trouve nulle trace de cet article dans la revue en question. Cette correspondance avec Espinas fait clairement apparaitre que Ribot n'était pas prêt à publier sous son nom, soit parce qu'il ne voulait pas être étiqueté comme un membre de l'école positiviste placé sous la bannière de Littré, soit parce qu'il craignait les foudres du clergé, mais la première hypothèse semble la plus probable, comme on le verra par la suite. À la fin de l'année 1868, il quitte Vesoul et est nommé à Laval, dans l'ouest de la France. C'est à l'époque une ville de 32000 habitants, plus près de la ville natale de Ribot, Guingamp, qui se trouve au nord de la Bretagne. Mais,

Voici le revers: [...] un Évêque, trois séminaires, 5 paroisses et le reste à proportion ; une nuée de prêtres, de jésuites. Un pays pourri de catholicisme. Des élèves nullement déniaisés, bien inférieurs à ceux de l'Est [...], dont les parents catholiques lisent et jugent mon cours.

Le niveau intellectuel est excessivement bas. Tout ce qui n'est pas fabricant de toile ou prêtraille n'est rien ici. [...] Le pays n'est hospitalier que pour les prêtres. ${ }^{15}$

Ribot fait état à plusieurs reprises, dans la suite de sa correspondance avec Espinas, des accusations d'athéisme portées contre lui par les parents catholiques de ses élèves. Cette correspondance met en évidence, bien mieux qu'un long exposé, la surveillance exercée sur les professeurs de philosophie dans la France du Second Empire et les pressions qu'ils subissaient, tant de la part du clergé que de la société civile.

9 Finalement, en 1872, pour terminer la traduction des Principes de psychologie, Ribot s'adjoindra Alfred Espinas, mais ce dernier semble avoir mis moins d'ardeur au travail qu'il ne l'escomptait et, finalement, le premier volume de l'ouvrage de Spencer ne 
paraîtra qu'en janvier 1874 et le second en novembre ${ }^{16}$ de la même année (mais il sera daté de 1875) chez l'éditeur Germer Baillière ${ }^{17}$. Entre-temps, en dépit de la guerre francoprussienne et de la Commune de Paris que, vue de Laval, Ribot juge "absurde», il publiera son premier ouvrage et soutiendra sa thèse.

En 1870, en effet, Ribot publie la Psychologie anglaise contemporaine, école expérimentale, chez l'éditeur Ladrange. Il présente longuement James Mill ainsi que John Stuart Mill, Herbert Spencer, Alexander Bain et Georges Lewes et consacre de courts chapitres à trois auteurs qu'il juge mineurs, Samuel Bailey, J. D. Morell et J. J. Murphy. En ce qui concerne Stuart Mill, rappelons que Taine lui avait déjà consacré un article paru dans la Revue des Deux Mondes en 1861, article qu'il avait remanié et publié en 1864 sous le titre Le Positivisme anglais. Étude sur Stuart Mill ${ }^{18}$.

11 Dès le début du chapitre qu'il consacre à Stuart Mill dans son ouvrage, Ribot s'efforce de dissiper la confusion, qu'il déplore, entre positivisme et esprit positif. En effet, le positivisme, doctrine d'Auguste Comte et de ses disciples, est selon lui une forme particulière de «l'esprit scientifique moderne » qui n'admet comme objet de science que "ce qui peut être observé, comme fait, ou formulé, comme loi, et vérifié ${ }^{19}$. Entre le positivisme et l'esprit positif, il trouve «autant de différences qu'entre l'esprit philosophique et la philosophie, c'est-à-dire entre ce qui demeure et ce qui passe $»^{20}$ et il tient le positivisme pour si catégorique et si dogmatique «qu'il impose plus aux esprits que la méthode moins affirmative de l'esprit purement scientifique $»^{21}$; on voit donc que Ribot s'emploie à dissiper un malentendu qu'il attribue peut-être au titre de l'étude de Taine. On voit surtout qu'il se démarque très fermement des positivistes français, ce qui se confirme dans le chapitre qu'il consacre à Spencer, qui s'ouvre et surtout se clôt sur une comparaison entre ce dernier et Auguste Comte. Car Spencer, esprit original et indépendant, appartient selon Ribot à l'espèce des «créateurs, qui par la puissance, la profondeur et l'unité de leur pensée, apparaissent, dès qu'on s'en approche, comme des hommes d'une autre famille $»^{22}$ que celle à laquelle appartiennent les «talents de second ordre ». Il ajoute, s'appuyant sur une citation de Stuart Mill, que comparé à Auguste Comte, Spencer n'est pas un disciple, mais un maitre et qu'il a repris scientifiquement à son compte l'idée de progrès, pure hypothèse chez Leibniz et "conception toute métaphysique » chez Hegel ${ }^{23}$. Chez Spencer, dit-il, l'idée de progrès surgit de l'observation des faits et de l'étude des sciences. La doctrine des correspondances entre l'organisme vivant et son milieu, enfin, lui parait une traduction «dans le langage de la psychologie expérimentale » de la monadologie leibnizienne ${ }^{24}$.

Le chapitre est ensuite consacré à un exposé de la loi d'évolution et de son application à la psychologie, exposé dont Spencer louera la qualité synthétique et la clarté, et il se termine par une comparaison entre Auguste Comte et Herbert Spencer, reprise d'un opuscule publié dès 1864 par Spencer lui-même ${ }^{25}$, à l'occasion de la parution en France, dans la Revue des Deux Mondes, d'un article sur ses Premiers principes ${ }^{26}$, dû à Auguste Laugel (1830-1914), polytechnicien, philosophe et historien. Ce dernier faisait de Spencer un héritier du positivisme comtien qui n'avait pas, cependant, rompu avec la métaphysique ${ }^{27}$ , ce qui était porté à son crédit. Ribot souligne que les points d'accord entre Spencer et Comte sont partagés par bien d'autres philosophes et que, en revanche, Spencer est en désaccord avec les points spécifiques à la doctrine de Comte. Il va de soi que Ribot ne pouvait admettre que Comte ait exclu la psychologie du cercle des sciences positives en raison de sa méthode d'observation interne et il souligne qu'« une moitié des Principes de psychologie est consacrée à une analyse subjective ${ }^{28}$. 


\section{particulier par Caro, Paul Janet et Lachelier, mais, écrivit-il à Espinas, «on trouve} cependant l'introduction "hardie" "subversive" positiviste d'allure » $(s i c)^{29}$.

\section{L'Hérédité psychologique, une thèse spencérienne}

En 1873, Ribot soutient ses deux thèses : d'abord une thèse latine sur Hartley, c'est-à-dire sur un «psychologue anglais» qui n'apparaît pas dans la $1^{\text {re }}$ édition de La Psychologie anglaise contemporaine, mais qu'il introduira dans la $2^{\mathrm{e}}$ édition, en 1875. Hartley (1705-1757) est certes un philosophe du XVIII ${ }^{\mathrm{e}}$ siècle, mais il y est placé en position de précurseur de l'associationnisme et de la psychologie physiologique du XIX ${ }^{e}$ siècle.

Sa thèse française s'intitule L'Hérédité: étude psychologique sur ses phénomènes, ses lois, ses causes, ses conséquences (publiée chez Ladrange la même année) ${ }^{30}$. Pourquoi l'hérédité ? Rappelons que, durant la seconde moitié du XIX $x^{e}$ siècle, la question de l'hérédité est devenue un thème récurrent. Selon Jean Borie, la Révolution française, qui détruit les privilèges héréditaires, fait passer la question de l'hérédité du droit à la biologie. Il s'agit de penser un mécanisme qui reproduit à l'identique et qui, en même temps, produit des différences ${ }^{31}$. C'est cette question-même que Ribot mettra au centre de sa thèse. Depuis les années 1850, à la suite, notamment, de la parution du Traité philosophique et physiologique de l'hérédité naturelle, du Dr Prosper Lucas ${ }^{32}$, l'hérédité est inscrite dans l'ordre biologique. Lucas justifie « scientifiquement » l'hérédité « des droits et des biens » par l'hérédité biologique :

[...] c'est du fait vital que le fait social procède [...] à nos yeux ce rapport est celui de la cause à l'effet: la cause, c'est la nature, l'effet l'institution [...] l'hérédité de nature devient en un mot, pour nous, la raison primordiale et la source réelle de l'hérédité d'institution. ${ }^{33}$

Ribot ne reprend pas à son compte ces thèses de Lucas, mais il cite largement les exemples trouvés chez cet auteur, de même qu'il cite les exemples d'hérédité morbide qu'il trouve chez les théoriciens de la folie héréditaire, comme Bénédict-Augustin Morel, Jacques-Joseph Moreau de Tours ou encore Jules Baillarger ${ }^{34}$ et tout au long de l'ouvrage il cite beaucoup d'auteurs français et étrangers, en particulier Galton et Darwin, ce qui montre l'importance du thème de l'hérédité à l'époque. Toutefois, comme l'écrira Taine, dans le compte-rendu de l'ouvrage de Ribot qu'il écrivit pour le Journal des Débats, dans tout l'ouvrage, «Spencer est son guide préféré " $^{35}$. Taine n'aimait guère Spencer, comme le montre la lettre qu'il écrit à Ribot pour le remercier de lui avoir envoyé sa thèse : s'il le félicite chaudement pour son travail, il regrette cependant son

[...] approbation presqu'absolue de toutes les idées de M. Herbert Spencer [...] ses Principles of Psychologie et ses First Principles sentent beaucoup trop, à mon avis, le métaphysicien ; il est jusqu'au cou dans l'hypothèse, expliquant toujours, non pas comment, en fait, les choses se font, mais comment il est possible qu'elles se fassent. ${ }^{36}$

En effet, s'il s'agit pour Ribot de montrer que "L'hérédité est la loi ", citation reprise à Darwin, à laquelle il ajoute que "la non hérédité est l'exception $»^{37}$, donc si l'hérédité explique la permanence, il faut aussi expliquer les variations. C'est là qu'intervient la loi d'évolution. Ribot souligne en premier lieu qu'il faut préférer le terme d'évolution ou de développement à celui de progrès, car « le progrès humain n'est qu'une partie du progrès total $\aleph^{38}$ et qu'avec le terme d'évolution, «le progrès n'apparaît plus comme la loi de 
l'humanité seule, mais comme la loi de la nature $»^{39}$. La loi d'évolution, que Spencer développe si magistralement dans les Premiers principes, écrit-il, qui repose sur «"une interprétation purement physique" des phénomènes, offre un caractère scientifique que n'a pas la doctrine courante du progrès $\aleph^{40}$. De plus, l'évolution finit, après avoir atteint l'équilibre pendant un temps de durée variable, par une période de dissolution, ce qui permet de rendre compte du fait que l'humanité ne semble pas toujours progresser et qu'elle retourne parfois en arrière. Selon Ribot, «cette loi domine toute la question des conséquences de l'hérédité $»^{41}$ car

L'évolution amène des modifications psychologiques et physiologiques; l'habitude les fixe dans l'individu, l'hérédité les fixe dans la race. Ces modifications accumulées et à la longue devenues organiques, rendent possible des modifications nouvelles, dans la suite des générations : ainsi l'hérédité devient en quelque sorte une puissance créatrice. ${ }^{42}$

Par conséquent, l'homme à la naissance n'est ni la tabula rasa des empiristes ni la statue de Condillac des sensualistes, puisqu'il hérite des acquis des générations précédentes, mais il peut aussi hériter de leurs pertes et, familles ou "races", sombrer dans la décadence. Car Ribot tient, à la suite de Taine, qu'il existe des caractères nationaux, lesquels sont selon lui d'abord dus à l'hérédité bien plus qu'aux institutions. Il affirme : «Le caractère national est l'explication dernière, la seule vraie, des vices et des vertus d'un peuple, de sa bonne et de sa mauvaise fortune. ${ }^{43}$ et « le Français du xix ${ }^{e}$ siècle est au fond le gaulois de César $»^{44}$. Il consacre un chapitre au caractère national et prend comme exemples, pour les opposer, deux «races», les juifs et les bohémiens. Dans les éditions suivantes de son ouvrage, considérablement remanié dès la deuxième édition, il explicitera le choix de ces deux « races » : les juifs, selon lui, « représentent le type le plus anciennement civilisé qui existe en Europe » tandis que les bohémiens «sont les plus réfractaires à la civilisation $»^{45}$. Ces deux groupes ayant en commun, dit-il, d'être restés relativement «fermés », ils sont supposés fournir des exemples de caractères nationaux primitifs et mettre en évidence, mieux que d'autres, l'action de l'hérédité. Il faut admettre que la thèse française $d u$ "grand ancêtre » de la psychologie française, présentée par Serge Nicolas ${ }^{46}$ comme "La première thèse française de psychologie "scientifique" ", nous fournit une éclatante illustration du racialisme de son époque. Même si Ribot met fermement en doute la doctrine de Gobineau (1853-1855) ${ }^{47}$ selon laquelle le métissage entraînerait la dégradation de la «race» blanche, et s'il condamne son apologie des « races pures », il ne doute pas un seul instant de la supériorité de la «race» blanche. Plus généralement, dans l'ensemble de l'ouvrage, il s'appuie sur une accumulation de «faits» dont il ne questionne pas la véracité : récits de voyageurs, témoignages ou opinions de missionnaires etc., qui lui permettent, pour ne prendre qu'un exemple, d'affirmer que des enfants philippins ou chinois, élevés en Europe ou dans un autre pays " civilisé ", reprennent aussitôt les habitudes de leurs « races " s'ils sont remis dans leur pays d'origine, quand ils ne prennent pas la fuite pour y retourner et reprendre leur vie primitive $^{48}$. Enfin, toute son argumentation repose sur l'hypothèse de l'hérédité des caractères acquis, sans laquelle la loi d'évolution ne pourrait produire ses effets bénéfiques. En effet, par exemple :

La constitution moyenne de l'esprit français aux sixième et neuvième siècles ne le rendait capable que d'un certain degré de culture : au-delà, il ne comprenait rien, défigurait tout, à la façon du sauvage zélandais. Mais cette constitution moyenne, améliorée par la culture, est léguée à la génération suivante, intérêts et capital, par celle-ci à une autre, et ainsi pendant dix ou douze siècles. ${ }^{49}$ 

lettres que Ribot adresse à Espinas, on en fit « une affaire d'état ». Elme Caro aurait, écritil, qualifié la thèse de "provocation en 600 pages $»^{52}$. Elle suscita davantage de remous encore après la soutenance. Tout d'abord, les quotidiens Le Temps (l'ancêtre du journal Le Monde) et La République française (journal fondé en 1871 par Gambetta) en firent des comptes-rendus élogieux. Le chroniqueur de La République française, Paul-Armand Challemel-Lacour se félicita de la soutenance de cette « œuvre de science » et proclama qu'« Au moment où le cléricalisme veut mettre la main sur la France, il est bon que les droits de l'investigation scientifique trouvent une forteresse dans le haut enseignement de l'Université. ${ }^{53}$ Comme on l'a vu, Taine présenta l'ouvrage dans le Journal des débats ; un jeune philosophe, Fernand Papillon, en fit un long compte-rendu dans la Revue bleue $e^{54}$ et il y eut plusieurs autres articles. Enfin, en novembre 1973, elle déclencha ce que Ribot qualifia d'« orage épouvantable $»^{55}$ à l'Académie des sciences morales et politiques. Le compte-rendu de la séance parut en décembre 1873 dans le Journal officiel et dans Le Temps et en 74 dans les comptes rendus des Séances et travaux de l'Académie: Caro, qui siégeait dans le jury de thèse de Ribot, présenta son ouvrage en soulignant qu'« Il ne s'agit plus de l'hérédité physiologique, il s'agit d'étendre la transmission héréditaire aux aptitudes et phénomènes de l'esprit, de la conscience, de la liberté. ${ }^{56}$ Comme on pouvait s'y attendre, Caro se montra pour le moins réticent vis-à-vis des conclusions de Ribot dont il jugea que l'esprit était " peut-être supérieur à son œuvre ${ }^{57}$ et conclut par l'affirmation que, du fait des questions non résolues par l'auteur, le spiritualisme n'était " pas même entamé »"58. La réaction d'un des membres de l'Académie, Adolphe Franck, est très intéressante : Adolphe Franck (1809-1893) était juif et titulaire de la chaire de droit de la nature et des gens au Collège de France et il proteste vigoureusement contre l'idée même d'hérédité, surtout dans les races, et rappelle que la «race sémitique [...] qu'on a dite héréditairement impropre à la philosophie ", a produit Spinoza ${ }^{59}$ et, plus généralement, il s'élève contre l'idée répandue (et reprise par Ribot dans sa thèse) selon laquelle les juifs sont héréditairement dénués de talents artistiques alors qu'ils ont montré, au cours de l'histoire, qu'ils les possèdent au plus haut degré, de même que toutes les autres aptitudes.

L'ouvrage de Ribot n'en eut pas moins de onze éditions en France jusqu'en 1925 et dix éditions aux États-Unis. Manifestement, sa thèse acheva de l'installer dans le paysage intellectuel français : en 1876, l'éditeur Germer Baillière fonda la Revue philosophique de la France et de l'Étranger dont Ribot prit la direction et la garda jusqu'à sa mort. Cette naissance fut commentée dans $L a$ Revue positive de Littré avec beaucoup de réserves. 
L'auteur de l'article, Georges Wybouroff (cofondateur et codirecteur de la revue avec Littré), reprocha à Ribot le ton trop conciliant de son programme et annonça que rien de bon ne pouvait sortir d'un tel œcuménisme car, écrivit-il, « le temps de l'éclectisme est passé $\aleph^{60}$. Il est clair que les positivistes français considéraient que Ribot était passé à l'ennemi.

Cette réaction complète un tableau qui montre que Ribot tenait à rester à égale distance des spiritualistes et des positivistes. En effet, être spencérien ne conduisait pas à s'aliéner les spiritualistes, qui pouvaient voir en Spencer une version acceptable d'un positivisme qui laissait une place à la métaphysique, grâce à la doctrine de l'Inconnaissable, encore qu'ils l'aient examinée avec beaucoup de méfiance. Et, d'un autre côté, même si les positivistes français rejetaient l'évolutionnisme et la métaphysique spencérienne, ils pouvaient estimer que Ribot était malgré tout du côté du positivisme. De plus, la doctrine spencérienne lui permettait de renvoyer dos à dos le matérialisme et le spiritualisme, pour défendre une psychologie débarrassée de la notion métaphysique de substance, comme il l'avait revendiqué dans son Introduction à La Psychologie anglaise contemporaine ${ }^{61}$.

Ribot continua de diffuser l'œuvre de Spencer en France, par l'intermédiaire de sa revue, qui publia vingt articles de Spencer entre 1876 et 1881 et autant de comptes rendus d'ouvrages de et sur Spencer. Toutefois, il semble qu'à la fin du siècle, il a, comme beaucoup d'autres, pris ses distances avec ce dernier, à la suite des critiques de Renouvier, en premier lieu, puis de Durkheim et de jeunes philosophes comme André Lalande et Gaston Richard. En effet, en 1901, Ribot fut chargé du rapport sur le « Concours pour le prix Crouzet » par l'Académie des Sciences Morales et Politiques. Parmi les quatre mémoires déposés, il soutint celui de Gaston Richard, anti-évolutionniste et antispencérien. L'Académie avait proposé le sujet suivant: L'idée d'évolution dans la nature et dans l'histoire. Et Ribot cita, sans trouver à y redire, les conclusions du mémoire "L'idée d'évolution ne peut jouer dans la science et la philosophie qu'un rôle négatif. » et « La théorie évolutionniste a réussi, moins par sa valeur scientifique que par la satisfaction qu'elle donne à l'esprit métaphysique $»^{62}$. Il conclut son rapport en indiquant que la section de philosophie avait proposé, "à l'unanimité » ${ }^{63}$, de décerner le prix Crouzet à l'auteur de ce mémoire.

\section{Conclusion}

Ainsi, il semble que son ralliement à l'évolutionnisme spencérien a eu deux fonctions pour Ribot. Tout d'abord, il lui a permis de contourner le positivisme français, en se revendiquant d'un autre positivisme, représenté par Spencer mais également par Stuart Mill. Ensuite, grâce à la métaphysique spencérienne, il a pu éviter, dans une certaine mesure, de s'aliéner totalement les spiritualistes, du moins ceux qui se disaient ouverts à la science.

Peut-on, pour autant, dire qu'il n'y eut qu'un «moment » spencérien de Ribot et que ce moment fut purement stratégique ? Certainement pas. Comme on l'a vu, il éprouva une très grande et très sincère admiration pour Spencer. Et la méthode pathologique qu'il mit au fondement de la psychologie qu'il appelait expérimentale, ainsi que ses premiers ouvrages et, en particulier, Les Maladies de la mémoire ${ }^{64}$, publié en 1881, furent marqués par un évolutionnisme d'origine spencérienne, revisité par le neurologue anglais John Hughlings Jackson, qui lui fournissait une version plus scientifique de la loi d'évolution, puisque fondée sur l'anatomie et la physiologie nerveuse ${ }^{65}$. Mais, pour autant qu'on puisse 
en juger, Ribot semble avoir, au cours des années 1880, pris ses distances vis-à-vis de la doctrine spencérienne. À notre connaissance, il ne s'en est jamais clairement expliqué, mais il a laissé quelques indices. En effet, en 1885, dans la leçon d'ouverture de son cours à la Sorbonne, intitulé «La psychologie nouvelle», il se félicite de l'essor de la psychologie animale et fait à ce propos l'éloge de l'idée d'évolution ${ }^{66}$, mais il ne cite pas Spencer. En revanche, il mentionne les travaux de John Lubbock, de Darwin, de George John Romanes et d'Espinas. Trois ans plus tard, en 1888, dans la leçon inaugurale de son cours au Collège de France, intitulée cette fois "La psychologie contemporaine", il consacre un développement à la psychologie anglaise, sujet de son célèbre ouvrage de 1870 , et déclare : «Je ne dirai rien des grands psychologues de ce pays, dont plusieurs, quoique vivants, nous apparaissent déjà dans la perspective de l'histoire. J'ai promis d'être strictement contemporain et je tiendrai ma promesse. $\aleph^{67}$ Là encore, il présente les recherches de Lubbock et de Romanes, et ne mentionne Spencer (qui manifestement, pour Ribot, appartient déjà à l'histoire) que pour signaler l'insuffisance de son hypothèse sur l'origine de l'instinct. Il se peut que, dès cette époque, Ribot ait pris en compte les critiques de Taine - qu'il admirait beaucoup - vis-à-vis de Spencer et rejeté, comme par trop métaphysique, l'hypothèse d'une loi d'évolution régissant le «développement universel ", comme il l'exprime à la suite de Gaston Richard dans son rapport de $1901^{68}$. Il n'est toutefois pas du tout certain qu'il ait abandonné «l'idée d'évolution " ${ }^{69}$ appliquée aux organismes vivants, qu'il avait placée aux fondements de la psychologie comparée. Quoi qu'il en soit, l'évolutionnisme spencérien, qui se trouve à la base du modèle neurologique de Jackson repris à son compte par Ribot, structure également la théorie de la hiérarchie des tendances de Pierre Janet, ainsi que, dans le domaine de la psychiatrie, l'approche organo-dynamique du psychiatre Henri Ey (1900-1977) ${ }^{70}$. Ainsi, le moment spencérien, en France comme ailleurs, a marqué durablement le domaine des sciences du psychisme.

\section{NOTES}

1. Cf. Daniel Becquemont et Laurent Mucchielli, Le Cas Spencer : religion, science et politique, Paris, PUF, 1998 ; Daniel Becquemont et Dominique Ottavi (dir.), Penser Spencer, Saint Denis, Presses Universitaires de Vincennes, 2011.

2. Henri Michel, «Herbert Spencer et Charles Renouvier ", L'Année psychologique, n 10, 1903, p. $142-160$, ici p. 146.

3. Hippolyte Taine, Les Philosophes français du XIX siècle, Paris, Hachette, 1857. Taine écrit dans la préface de cet ouvrage que le spiritualisme est une philosophie "à l'usage des lettrés » et le positivisme une philosophie "à l'usage des savants» (p. IV). Pour un ouvrage récent sur la question, voir Jean Lefranc, La Philosophie en France au XIX siècle, Paris, L'Harmattan, 2011 [1998].

4. Jean-Paul Cointet, Hippolyte Taine, un regard sur la France, Paris, Perrin, 2012.

5. Victor Hugo contre la loi Falloux (janvier 1850). Document en ligne, novembre 2012, http:// clioweb.free.fr/textes/hugo1850.htm (consulté le 2 avril 2014).

6. Victor Duruy (1811-1894), historien et homme politique français. En 1863, au moment de la libéralisation de l'Empire, il fut nommé ministre de l'Instruction publique par Napoléon III. Il 
rétablit notamment l'agrégation de philosophie (l'agrégation d'histoire avait été rétablie dès $1860)$ et il fit voter une loi obligeant toutes les communes de plus de 500 habitants à créer une école de filles.

7. Raymond Lenoir, «Lettres de Théodule Ribot à Espinas ", Revue philosophique de la France et de l'Étranger, n 147, 1957, p. 1-14, ici p. 1. Les lettres envoyées par Ribot à Espinas, de 1866 à 1893, ont été partiellement publiées par Raymond Lenoir, dans cinq volumes de la Revue philosophique, entre 1957 et 1975 ; Alfred Espinas (1844-1922) fut un philosophe et sociologue français, qui soutint, en 1877, sa thèse intitulée Des Sociétés animales, dont la pertinence philosophique fut contestée par le jury, composé de spiritualistes. De fait, l'ouvrage d'Espinas était clairement antispiritualiste. La thèse, précédée d'une introduction que son auteur avait dû supprimer pour la soutenance, fut publiée en 1877 également (Alfred Espinas, Des Sociétés animales, étude de psychologie comparée, Paris, Germer Baillière, 1877). Voir Wolf Feuerhahn, «Les "sociétés animales" : un défi à l'ordre savant ", Romantisme, n 154, Paris, Armand Colin, 2011, p. 35-51.

8. Ibid.

9. Ibid., p. 2.

10. Ibid.

11. Ibid. ; souligné par Ribot.

12. Souligné par moi.

13. Ibid., p. 3.

14. Ibid.

15. Ibid., p. 4 ; les passages en italiques sont soulignés par Ribot.

16. Dans une lettre à Espinas, datée du 23 novembre 1874, Ribot lui annonce que le deuxième volume des Principes de psychologie vient de paraitre (Lenoir, "Lettres de Théodule Ribot à Espinas ", op. cit., p. 13).

17. Herbert Spencer, Principes de psychologie (2 vol.), trad. Th. Ribot et A. Espinas sur la seconde édition anglaise (1870-1872), Paris, Germer Baillière, 1874-1875.

18. Hippolyte Taine, "Philosophie anglaise. John Stuart Mill et son système de logique ", Revue des Deux Mondes, $\mathrm{n}^{\circ}$ 32, 1861, p. 44-82, et Le Positivisme anglais. Étude sur Stuart Mill, Paris, Germer Baillière, 1864.

19. Théodule Ribot, La Psychologie anglaise contemporaine (École expérimentale), Paris, Ladrange, 1870 , p. 89.

20. Ibid., p. 90.

21. Ibid.

22. Ibid., p. 145.

23. Ibid., p. 148.

24. Ibid., p. 150.

25. Herbert Spencer, The Classification of the Sciences : to Which Are Added Reasons for Dissenting from the Philosophy of M. Comte, New York, D. Appleton \& Co., 1864.

26. Les Premiers principes de Spencer étaient parus en langue anglaise en 1862.

27. Auguste Laugel, «Les études philosophiques en Angleterre. M. Herbert Spencer », Revue des Deux Mondes, $\mathrm{n}^{\circ} 49,1864$, p. 930-957. Dans cet article, Laugel qualifie Spencer de «dernier métaphysicien anglais » (p. 934) et écrit à propos du positivisme spencérien « Cette doctrine, qui séduit aujourd'hui beaucoup d'esprits, a été une réaction contre la métaphysique, et cependant elle est obligée d'emprunter quelque chose à son ennemie » (p. 957).

28. Ibid., p. 220.

29. Lenoir, «Lettres de Théodule Ribot à Espinas », op. cit., p. 5.

30. Théodule Ribot, L'Hérédité, étude psychologique sur ses phénomènes, ses lois, ses causes, ses conséquences, Paris, Ladrange, 1873.

31. Jean Borie, Mythologies de l'hérédité au XIX siècle, Paris, Éditions Galilée, 1981. 
32. Prosper Lucas, Traité philosophique et physiologique de l'hérédité naturelle dans les états de santé et de maladie du système nerveux (2 vol.), Paris, J.-B. Baillière, 1847-1850.

33. Ibid., vol. 1, p. 5. Les italiques sont de Prosper Lucas.

34. Jean-Christophe Coffin, La Transmission de la folie. 1850-1914, Paris, L'Harmattan, 2003.

35. Hippolyte Taine, "L'Hérédité, étude psychologique, par Charles [sic] Ribot ", Journal des débats politiques et littéraires, 23 novembre 1873, p. 3.

36. Hippolyte Taine, «Lettre du 6 juillet 1873. À M. Théodule Ribot», H. Taine. Sa vie et sa correspondance, tome III, L'Historien (1870-1875), Paris, Hachette, 1905, p. 237-239, ici p. 238.

37. Ribot, L'Hérédité, étude psychologique sur ses phénomènes, ses lois, ses causes, ses conséquences, op. cit., p. 201.

38. Ibid., p. 396.

39. Ibid.

40. Ibid., p. 399.

41. Ibid., p. 400.

42. Ibid., p. 400-401.

43. Ibid., p. 151-152.

44. Ibid., p. 154. Selon Ribot, les « traits essentiels de notre caractère national » seraient « l'amour des armes, le goût de tout ce qui brille, l'incroyable légèreté d'esprit, la vanité incurable, la finesse, une grande facilité à parler et à se laisser prendre par les mots » (ibid.).

45. Théodule Ribot, L'Hérédité psychologique, Paris, Alcan, 1894, p. 127.

46. Serge Nicolas, «L'hérédité psychologique d'après Théodule Ribot (1873). La première thèse française de psychologie "scientifique" ", L'Année psychologique, n 99, 1999, p. 295-348, ici p. 295.

47. Arthur de Gobineau, Essai sur l'inégalité des races humaines (4 vol.), Paris, Firmin-Didot frères, 1853-1855.

48. Ribot, L'Hérédité, étude psychologique sur ses phénomènes, ses lois, ses causes, ses conséquences, op. cit., p. 464-466.

49. Ibid., p. 451.

50. Cf. August Weismann, Essais sur l'hérédité et la sélection naturelle, Paris, C. Reinwald, 1892, trad. Henry de Varigny.

51. Raymond Lenoir, «Lettres de Théodule Ribot à Alfred Espinas (1876-1893)", Revue philosophique de la France et de l'Étranger, n 165, 1975, p. 165-172, ici p. 171. Notons qu'un recueil des travaux de Weismann avait été publié en français en 1892.

52. Lenoir, «Lettres de Théodule Ribot à Espinas », op. cit., p. 10.

53. Cité par Serge Nicolas, «L'hérédité psychologique d'après Théodule Ribot (1873). La première thèse française de psychologie "scientifique" ", op. cit., p. 319.

54. Fernand Papillon, «Thèses de M. Th. Ribot: L'association des idées dans Hartley. L'hérédité psychologique ", La Revue politique et littéraire, $\mathrm{n}^{\circ}$ 11, 1873, p. 1214-1218.

55. Lenoir, « Lettres de Théodule Ribot à Espinas », op. cit., p. 12.

56. Séances et travaux de l'Académie des sciences morales et politiques, $\mathrm{n}^{\circ} 1$ (Nouvelle Série), $33^{\mathrm{e}}$ année, Paris, 1874, p. 536-540.

57. Ibid., p. 536.

58. Ibid., p. 538.

59. Ibid., p. 539.

60. Georges Wibouroff, "Revue philosophique de la France et de l'Étranger », La Philosophie positive, $\mathrm{n}^{\circ} 16,1876$, p. 468-469.

61. Op. cit., p. 30.

62. Théodule Ribot, « Rapport sur le concours pour le prix Crouzet à décerner en 1901. L'idée de l'évolution dans la nature et dans l'histoire ", Séances et travaux de l'Académie des sciences morales et politiques, $\mathrm{n}^{\circ}$ 56, 1901, p. 643-644.

63. Ibid., p. 644 , souligné par moi. 
64. Théodule Ribot, Les Maladies de la mémoire, Paris, Germer Baillière, 1881.

65. Jacqueline Carroy, Annick Ohayon et Régine Plas, Histoire de la psychologie en France: $\mathrm{XIX}^{e}-\mathrm{XX} \mathrm{X}^{e}$ siècles, Paris, La Découverte, 2006.

66. «Grâce à cette idée d'évolution qui est l'âme de la psychologie comparée, les diverses manifestations mentales chez l'homme ne peuvent plus être traitées comme des faits sans analogues et sans précédents dans la nature, mais comme le dernier terme d'un progrès dont l'origine se confond avec l'origine même de la vie. » (« Psychologie expérimentale. Cours de M. Th. Ribot. Leçon d'ouverture. La psychologie nouvelle », La Revue politique et littéraire, $\mathrm{n}^{\circ} 36,1885$, p. 780-787, ici p. 786.) Notons que Ribot ne parle plus de la «loi d'évolution » mais de «l'idée d'évolution ».

67. Théodule Ribot, «Leçon d'ouverture du cours de psychologie expérimentale et comparée du Collège de France. La psychologie contemporaine », Revue scientifique (Revue rose), $\mathrm{n}^{\circ} 15$ (troisième série), 1888, p. 449-452.

68. Ribot, « Rapport sur le concours pour le prix Crouzet à décerner en 1901. L'idée de l'évolution dans la nature et dans l'histoire », op. cit., p. 639, souligné par moi.

69. «Psychologie expérimentale. Cours de M. Th. Ribot. Leçon d'ouverture. - La psychologie nouvelle », op. cit., souligné par moi.

70. Cf. Jean-Christophe Coffin (dir.), Conceptions de la folie et pratiques de la psychiatrie autour d'Henri Ey (Colloque de l'ENS des 14-15 septembre 2006), Les Cahiers Henri Ey, $\mathrm{n}^{\circ}$ 20-21, 2008.

INDEX

Mots-clés : psychologie, hérédité, Ribot (Théodule)

\section{AUTEUR}

\section{RÉGINE PLAS}

Université Paris Descartes 